\title{
Inclusion of information costs in process design optimization under uncertainty
}

\author{
Fernando P. Bernardo ${ }^{a, *}$, Pedro Saraiva a ${ }^{\text {a }}$ Efstratios N. Pistikopoulos ${ }^{b}$ \\ a Department of Chemical Engineering, University of Coimbra, Pólo II-Pinhal de Marrocos, 3030-290 Coimbra, Portugal \\ ${ }^{\mathrm{b}}$ Department of Chemical Engineering, Centre for Process Systems Engineering, Imperial College, London $S W 72 B Y, U K$
}

\begin{abstract}
Recent developments in process design have focused on establishing optimization-based approaches to support decision-making under uncertainty, but few efforts have been made to study and consider how information regarding this uncertainty affects optimal decisions. In this paper we develop an optimal design framework that, besides integrating process profitability, robustness and quality issues, allows one to decide how much it is worth to spend in research and experimentation for selectively reducing parameter uncertainties and guiding $R \& D$ activities. The design problem is thus formulated as a stochastic optimization problem, whose objective function includes an information cost term, leading to the identification of optimal parameter uncertainty levels one should end up with, as well as the corresponding amounts to be spent in R\&D. A case study comprising a reactor and heat exchanger system is introduced and provides an illustrative application for the suggested methodology. (C) 2000 Elsevier Science Ltd. All rights reserved.
\end{abstract}

Keywords: Stochastic process design; Uncertainty; Value of information; R\&D economics

\section{Introduction}

At the design stage of a chemical plant, decision making is frequently subject to different sorts of uncertainty. Equipment configuration, dimensions and operating conditions have to be decided on the basis of an available process model, whose parameters may be uncertain, and on external information (such as product demand), which commonly exhibits a random behavior.

Recent developments in process design have focused on establishing optimization-based frameworks that take into account different sources of variability (Pistikopoulos, 1995). Namely, several approaches have been proposed to formulate and solve process design problems under uncertainty, differing in how uncertainty is modeled, control variables adjusted in face of uncertainty, and design objectives (Bernardo, Pistikopoulos \& Saraiva, 1999b) considered (process flexibility, profitability, quality and/or robustness). However, few

\footnotetext{
* Corresponding author. Tel.: + 351-239-798722; fax: + 351-239798703.

E-mail address: bernardo@eq.uc.pt (F.P. Bernardo)
}

efforts have been made and reported in the literature about the ways parameter uncertainties affect optimal decisions and how this issue can be included in the process design problem formulations.

In an increasingly competitive environment, we believe that it is particularly relevant to explicitly address this issue for at least the two following main reasons: (1) due to both time and resource constraints, R\&D efforts for parameter estimation and uncertainty reduction have to be very selectively guided and managed according to cost-benefit analysis; (2) the need for very rapid product and process development, together with the strong interactions between design, operation and R\&D decisions, point clearly towards concurrent engineering approaches, where decisions regarding all of the above are taken simultaneously and preferably as the result of fully integrated approaches and problem formulations. The goal of this article is thus to provide a contribution in this area, by developing and testing a process design framework that takes into account information value and its associated costs, combined together with other cost components in order to achieve overall best design and $\mathrm{R} \& \mathrm{D}$ decisions. 
For addressing this question, we distinguish among two different categories of uncertain parameters: (U1), for uncertain parameters whose variability can be reduced namely through experimentation and R\&D; and (U2), standing for random parameters whose uncertainty reduction is believed to be beyond our control (one has to address and live with the present levels of uncertainty for these parameters). Uncertain process model parameters, such as a kinetic constant, fall in the first category, since information regarding them can be obtained, although at a certain cost (laboratory or pilot plant experiments, etc.). On the other hand, we will consider parameters such as a product demand to belong to the second category, assuming that their variability is due to market fluctuations that cannot be further reduced.

According to the above uncertainty categories, we then pose the following questions: (Q1) what is the optimal investment that should be selectively allocated to $R \& D$ activities, in order to reduce uncertainty in parameters (U1), which parameters in particular deserve special attention in this regard and what are the final expected uncertainty levels that one should get and live with; and (Q2) what is the loss of opportunity associated with a decision taken without having any additional information about parameters (U2).

Ierapetritou, Pistikopoulos and Floudas (1996) addressed question $\left(Q_{2}\right)$ defining mathematically the value of information regarding parameters (U2) as the difference between two behavioral models of decision making under uncertainty: design decisions taken prior to resolution of uncertainty (here-an-now model) versus decisions adjusted to uncertainty realizations (wait-and-see model).

Based upon our previous work on optimal design under uncertainty considering process robustness and quality criteria (Bernardo \& Saraiva, 1998; Bernardo, Pistikopoulos \& Saraiva, 1999a, 1999b), we propose here a process design framework that embeds question (Q1) within a stochastic optimization formulation. Besides investment, operating and quality costs, the objective function includes an information cost term, corresponding to the annual depreciation cost deriving from $R \& D$ investments associated with selective parameter uncertainty reduction. Therefore, the optimal design solution obtained will reflect, for each (U1) parameter, a trade-off between the benefits and economic value added by uncertainty reduction and the information and knowledge cost associated with obtaining such a reduction of uncertainty.

Before presenting the suggested methodology, we will first introduce a motivating example in order to clarify some of the ideas, concepts and notation used; then, we develop the corresponding generic process design mathematical formulation, which is then applied to the motivating example.

\section{Motivating example}

In order to clarify the meaning and relevance of question (Q1), we will now consider a simple flow sheet (Fig. 1), consisting of a reactor and heat exchanger, where a first order exothermic reaction $\mathrm{A} \rightarrow \mathrm{B}$ takes place (Halemane \& Grossmann, 1983; Bernardo et al. 1999b; Georgiadis \& Pistikopoulos, 1999, where its complete model can be found).

The initial uncertainties considered comprise six independent and normally distributed parameters (Table 1), with the last three clearly falling under category (U1), since their uncertainty can be reduced by running additional experiments.

Therefore, our process design problem formulation should allow us to decide a priori, besides the optimal values for reactor volume and heat exchanger transfer area, how much it is worth investing in $R \& D$ for reducing parameter (U1) uncertainties, to what extent this should be carried out and for which of the parameters, according to the overall economic gains that derive from obtaining additional information about each of them.

\section{Process design problem formulation}

The problem formulation that we will present here is based on a two-stage stochastic formulation previously

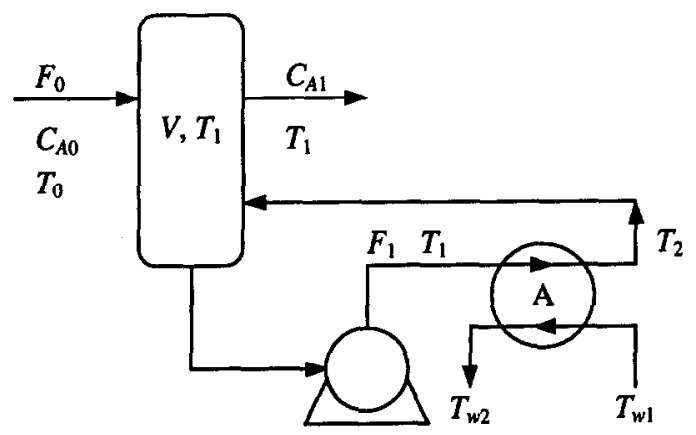

Fig. 1. Reactor and heat exchanger system.

Table 1

Initial parameter uncertainties

\begin{tabular}{lll}
\hline & Mean $(\mu)$ & Error $^{\mathrm{a}}(\varepsilon)$ \\
\hline$F_{0}$ & $45.36 \mathrm{kmol} \mathrm{h}^{-1}$ & 0.20 \\
$T_{0}$ & $333 \mathrm{~K}$ & 0.04 \\
$T_{w 1}$ & $293 \mathrm{~K}$ & 0.04 \\
$k_{R}$ & $12 \mathrm{~h}^{-1}$ & 0.30 \\
$U$ & $1635 \mathrm{~kJ} \mathrm{~m}^{-2} \mathrm{~h}^{-1} \mathrm{~K}^{-1}$ & 0.30 \\
$E / R$ & $555.6 \mathrm{~K}$ & 0.30 \\
\hline
\end{tabular}

${ }^{a}$ Standard deviation, $\sigma=\varepsilon \mu / 3.09$. 
Table 2

Problem (1) nomenclature

\begin{tabular}{|c|c|c|}
\hline & Description & Examples \\
\hline$d$ & Design variables (vector) & Equipment dimensions and configurations \\
\hline$z$ & Control variables (vector) & Process flow rates \\
\hline$x$ & State variables (vector) & Process temperatures \\
\hline$y$ & $\begin{array}{l}\text { Quality related variables (vector), with desired values } y^{*} \text {, } \\
\mu_{y} \text { means and } \sigma_{y} \text { standard deviations }\end{array}$ & Product stream composition \\
\hline$\theta$ & Uncertain parameters (vector) & Heat transfer coefficient, product demand \\
\hline$\Theta$ & Uncertainty space & \\
\hline$f_{q}$ & Objective function (scalar) & $\begin{array}{l}\text { Investment cost + operating cost + quality } \\
\text { cost }\end{array}$ \\
\hline$E$ & Expectancy operator & \\
\hline$h$ & Process model equations (vector) & $\begin{array}{l}\text { Heat and mass balances, equilibrium } \\
\text { relationships }\end{array}$ \\
\hline$g$ & Process model inequalities (vector) & Safety or environmental hard constraints \\
\hline
\end{tabular}

developed by Bernardo et al. (1999a,b), where process robustness issues and quality metrics are explicitly addressed, besides equipment and operating costs. Uncertainty is modeled as a probabilistic space of the form $\Theta=\{\theta: \theta \in j(\theta)\}$, where $j$ is a joint probability density function (PDF) for the random vector of uncertain parameters $\theta$. Design variables are considered to be 'here-and-now' decisions (design stage), while control variables are addressed as 'wait-and-see' decisions (operating stage). Process robustness is taken into account through the inclusion of a quality cost term (such as a Taguchi loss function) in the objective function and/or the assumption of explicit hard constraints over certain variables (e.g. product characteristic variance upper limit).

The above concerns can be mathematically described by the following two-stage optimization problem (nomenclature in Table 2), where as hard quality constraints we assume here a maximum upper value for the quality variables relative error (standard deviation to mean ratio):

\section{Design stage:}

$\min _{d} E_{\Theta}\left\{f_{q}^{\prime}(d, \theta)\right\}$

s.t. $\frac{\sigma_{y}}{\mu_{y}} \leq \gamma, \quad \mu_{y}=E_{\Theta}\left(y^{\prime}\right), \quad \sigma_{y}^{2}=E_{\Theta}\left\{\left(y^{\prime}-\mu_{y}\right)^{2}\right\}$

$d \in D, \quad \theta \in \Theta$

\section{Operating stage:}

$$
\begin{aligned}
& f_{q}^{\prime}(d, \theta)=\min _{z, x, y} f_{q}\left(d, z, x, y, y^{*}, \theta\right) \\
& \text { s.t. } h(d, z, x, \theta)=0 \\
& g(d, z, x, \theta) \leq 0 \\
& y^{\prime}=y \\
& z \in Z, \quad x \in X, \quad y \in Y
\end{aligned}
$$

In formulation (1), it is further assumed that all the constraints leading to unfeasibility in the inner optimization problem can be treated as quality soft constraints, and therefore such constraints are replaced by a corresponding Taguchi loss function, included in $f_{q}$. Thus, the expected value of a general scalar function $f$ is evaluated through an $n$-dimensional integral over the entire uncertainty space $\Theta$ ( $n$ is the number of uncertain parameters), that can be approximated by an integration formula with a grid of $N_{p}$ points $\theta_{i}$ and corresponding weights $w_{i}(2)$ :

$E_{\Theta}(f)=\int_{\Theta} f(\theta) j(\theta) d \theta \cong \sum_{i=1}^{N_{p}} w_{i} f\left(\theta_{i}\right)$

The number of points in the integration formula $\left(N_{p}\right)$ is a critical factor from a computational intensity point of view. As before (Bernardo, Pistikopoulos \& Saraiva, 1999c), in this article we will employ an efficient cubature formula, specially constructed for the situation where all uncertain parameters are normally distributed, leading to a total number of integration points $N_{p}=2^{n}+2 n$.

In order to embed question (Q1) within our previous problem formulation (1), we will now consider the subset $J_{1}$ of uncertain parameters whose variability can be further reduced through R\&D efforts (uncertain parameters belonging to category (U1)). The annual additional information cost, associated with the experiments needed to reduce uncertainty in parameter $\theta_{j}$, $j \in J_{1}$, is assumed to be given by a function of the form:

$C_{I, j}=b_{j} C_{i f, j}+\alpha_{j}\left(\frac{1}{\varepsilon_{j}}-\frac{1}{\varepsilon_{j}^{N}}\right)$

where $C_{I f j}$ represents a fixed cost (associated for instance with the investment in laboratory or pilot scale equipment needed to run the experiments), while the second term corresponds to variable costs (e.g. reactant and operation costs). If no experiment takes place, the binary variable $b_{j}$ affecting the fixed cost equals zero 
and the relative error associated with parameter $\theta_{j}$ remains at its current nominal level $\left(\varepsilon_{j}=\varepsilon_{j}^{N}\right)$. Otherwise, $b_{j}=1$ and the information cost grows as the relative error decreases, since more precise knowledge about $\theta_{j}$ becomes available; in the limit, an infinite R\&D investment would lead to perfect knowledge about this parameter.

The total cost of information is thus given by the following sum over $J_{1}$ :

$C_{I}=\sum_{j \in J_{1}} C_{I, j}$

The definition of $\varepsilon_{j}$ depends on the PDF used to describe uncertainty over parameter $\theta_{j}$. For instance, when $\theta_{j}$ is described by a normal PDF $\mathrm{N}\left(\mu_{j}, \sigma_{j}\right)$, we will consider $\varepsilon_{j}$ to be such that $p\left[\mu_{j}\left(1-\varepsilon_{j}\right) \leq \theta_{j} \leq \mu_{j}(1+\right.$ $\left.\left.\varepsilon_{j}\right)\right]=0.998$, i.e. $\sigma_{j}=\varepsilon_{j} \mu_{j} / 3.09$, and use this threshold to truncate normal PDFs.

When a certain positive amount $C_{I, j}$ is spent in R\&D, our knowledge about parameter $\theta_{j}$ increases, namely leading to a different and more accurate estimate for its true mean, with a reduced standard deviation, when compared with the current level of information available about this parameter.

However, for the sake of simplicity and illustrative purposes only, we will first consider a hypothetical situation where the true $\theta_{j}$ mean value is assumed to be known a priori.

\subsection{Parameter true mean values known a priori}

If one assumes that the parameters (U1) true mean values are known a priori, all $R \& D$ efforts are aimed at reducing standard deviation levels. The interaction between information costs and associated benefits due to uncertainty reduction can be explored by including an information cost term like (4) in the design objective function and considering the relative errors of uncertainties $\theta_{j}, j \in J_{1}$, as additional decision variables. Taking this into account, and using the integration formula (2) to estimate expected values in face of uncertainty, our formulation (1) becomes the following MINLP problem:

Table 3

Results with B and without A considering information cost terms

\begin{tabular}{lcc}
\hline & $\mathrm{A}$ & $\mathrm{B}$ \\
\hline$E$ cost (S per year) & 14596 & 13757 \\
$V\left(\mathrm{~m}^{3}\right)$ & 8.254 & 4.779 \\
$A\left(\mathrm{~m}^{2}\right)$ & 7.962 & 7.147 \\
$\varepsilon\left(k_{R}\right)$ & 0.300 & 0.172 \\
$\varepsilon(U)$ & 0.300 & 0.300 \\
$\sigma(E / R)$ & 0.300 & 0.137 \\
$\mu\left(x_{\mathrm{A}}\right)$ & 0.9421 & 0.9062 \\
$\sigma\left(x_{\mathrm{A}}\right) / \mu\left(x_{\mathrm{A}}\right)$ & 0.01 & 0.01 \\
\hline
\end{tabular}

$$
\begin{aligned}
& \min _{d, b_{j}, \varepsilon_{j}, z_{i}, x_{i}, y_{i}} \sum_{i=1}^{N_{p}} w_{i} f_{q}\left(d, z_{i}, x_{i}, y_{i}, y^{*}, \theta_{i}\right)+C_{I}\left(b_{j}, \varepsilon_{j}\right) \\
& \text { s.t. } 0 \leq \varepsilon_{j} \leq \varepsilon_{j}^{N} \\
& \varepsilon_{j}^{N}-\varepsilon_{j} \leq b_{j} \varepsilon_{j}^{N} \\
& \frac{\sigma_{y}}{\mu_{y}} \leq \gamma, \quad \mu_{y}=\sum_{i=1}^{N_{p}} w_{i} y_{i}, \quad \sigma_{y}^{2}=\sum_{i=1}^{N_{p}} w_{i}\left(y_{i}-\mu_{y}\right)^{2} \\
& h\left(d, z_{i}, x_{i}, \theta_{i}\right)=0 \\
& g\left(d, z_{i}, x_{i}, \theta_{i}\right) \leq 0 \\
& d \in D, \quad b_{j} \in\{0,1\}, \quad z_{i} \in Z, \quad x_{i} \in X, \quad y_{i} \in Y \\
& i=1, \ldots, N_{p} ; j \in J_{1}
\end{aligned}
$$

We have applied this problem formulation to our previous plant comprising a reactor and heat exchanger (Fig. 1), where the reactor volume, $V$, and the heat exchanger area, $A$, are the design variables, while flow rates in the heat exchanger, $F_{1}$ and $F_{w}$, are control variables optimally adjusted to uncertainty during the operating stage (variables indexed over $i$ ). The conversion of $\mathrm{A}$ in the reactor, $x_{\mathrm{A}}$, is the only quality variable, with a minimum acceptable value of $y^{*}=0.9$, and process robustness is taken into account through a quality cost term that penalizes only conversions smaller than 0.9 (one-sided Taguchi loss function): $C_{q}=k\left(x_{\mathrm{A}}-0.9\right)^{2}$, where $k=k_{1}=6.4 \times 10^{6}$ if $x_{\mathrm{A}}<0.9$ and $k=0$, otherwise (Bernardo et al., 1999a). Furthermore, a hard quality constraint on $x_{\mathrm{A}}$ was also incorporated into the problem formulation: $\sigma\left(x_{\mathrm{A}}\right) / \mu\left(x_{\mathrm{A}}\right) \leq 0.01$. The objective function also includes equipment, operating and information costs, with the information cost term computed considering all the three uncertain parameters, $k_{R}, U$ and $E / R$ (Table 1) that belong to category (U1), with initial nominal errors equal to 0.3 , fixed research costs equal to $100 \$$ per year and $\alpha=90$. Using a specialized cubature formula, this problem can be formulated as (5) and solved with 76 integration points in the uncertainty domain.

The results thus obtained (Table 3) allow one to compare scenario A, which corresponds to the optimal solution obtained under the initial level of uncertainty (Table 1), with scenario B, that corresponds to the best solution found for the proposed formulation (5), where optimal levels of uncertainty are decided based upon information benefit-cost analysis. For this particular hypothetical situation, there is a decrease in the overall expected cost of 839 \$ per year, showing that it is worthwhile spending a certain amount of resources in uncertainty reduction $\left(C_{I}=779 \$\right.$ per year $)$, specially concerning activation energy (where an optimal error of only 0.137 was identified), found to be the parameter where more intensive $\mathrm{R} \& \mathrm{D}$ efforts should be allocated; on the other hand, the present levels of knowledge about the heat transfer coefficient seem to be good 
enough, due to the low impact of further uncertainty reduction for $U$ in terms of overall expected plant cost.

By focusing optimal $R \& D$ decisions on the right targets, one knows how much to spend in different experiments for reducing uncertainty of relevant parameters around its true mean values (supposed here to be known a priori) and how much additional economic value is thus obtained. Next, we will consider the more generic, complex but realistic situation, where the true parameter means are not known.

\subsection{General parameter uncertainty formulation}

The assumption of a priori known true mean values for uncertain parameters (U1) is unrealistic, and therefore $R \& D$ efforts have in general to be conducted in order to reduce uncertainty levels for parameter variability around an unknown true mean value. Therefore, the optimal levels of uncertainty reduction should be decided taking into account all the possible true mean values for a certain parameter.

We address this more complex problem in two stages: first, we identify and screen those parameters of class (U1) whose uncertainty elimination does have a larger economic impact over average process economic performance (this analysis is performed considering each parameter at a time, and thus the effects of uncertainty interactions are neglected); then, for the reduced subset thus found, we define how much R\&D spending and which uncertainty reduction levels correspond to an optimal solution.

For the first stage, let us consider: parameter $\theta_{j}, j \in$ $J_{1}$, with an initial level of uncertainty described by the PDF $p\left(\theta_{j}\right) ; C_{1}$ to be the objective function value for the solution of problem (1) obtained considering present $\theta_{j}$ uncertainty levels; and $C_{2}$ the objective function value for the solution of the same problem but considering now that one knows exactly $\theta_{j}$ true value. Then, the economic value which corresponds to the complete elimination of uncertainty (VEU) for parameter $\theta_{j}$ is given by: $\mathrm{VEU}=C_{1}-C_{2}$; but since at the present level of knowledge $\theta_{j}$ can take any value in the $p\left(\theta_{j}\right)$ domain, the expected value for complete uncertainty elimination on $\theta_{j}$ becomes:

$E(\mathrm{VEU})=C_{1}-\int C_{2}\left(\theta_{j}\right) p\left(\theta_{j}\right) \mathrm{d} \theta_{j}$

The concept of VEU can be seen, similarly with what happens with the value of perfect information, VPI (Ierapetritou et al., 1996), as the difference between a here-and-now decision, corresponding to cost $C_{1}$, made under the present level of uncertainty, and a wait-andsee decision, corresponding to cost $C_{2}$. However, they refer to different kinds of uncertain parameters: while VPI refers to parameters of class (U2), with the waitand-see decision made after uncertainty realization,
VEU refers to parameters of class (U1), where the wait-and-see decision corresponds to uncertainty elimination. Referring to our earlier example (Fig. 1), we have estimated (6) for its three parameters of type (U1), using a specialized quadrature formula, leading to expected VEU values of 379,0 and $1279 \$$ per year, respectively for parameters $k_{R}, U$ and $E / R$. This reveals that activation energy is indeed the parameter whose uncertainty reduction is most relevant for achieving overall plant cost savings, and therefore $R \& D$ investmonts regarding this parameter seem to be particularly valuable. A closer look at this parameter can be made by plotting objective function values $C_{2}$ as a function of $E / R$ true but unknown value (Fig. 2). The mean value of this function, weighted by the normal PDF $p(E / R)$ ( $\mu=555.6 \mathrm{~K}, \varepsilon=0.3$ ) that corresponds to our present knowledge about this parameter, is $13317 \$$ per year, and therefore the expected VEU for $E / R=1279 \$$ per year, (14 596, objective function value $C_{1}$, minus $13317)$.

Once we have selected those parameters whose uncertainty reduction seems to have a significant impact over process economic performance (according to their expected VEU), we focus R\&D efforts around this subset and now, on a second stage, try to identify the optimal investments that should be made for each of them, together with the corresponding uncertainty levels that we will end up with for such parameters.

We will thus call $J_{2}$ the group of screened value adding parameters, over which R\&D cfforts should focus, with an initial level of uncertainty described by the joint PDF $p\left(\theta_{j}\right)$, with vector of means $\mu_{j}$ and relative errors $\varepsilon_{j}, j \in J_{2}$. When a certain amount $C_{I}$ is spent in order to reduce the uncertainty currently associated with such parameters, vector $\mu_{j}$ can take any value in the domain of $p\left(\theta_{j}\right)$ for unknown true mean values, and the errors $\varepsilon_{j}$ are reduced according to cost function (3), depending on the magnitude of investments to be made as information costs.

In order to find out what are the optimal errors $\varepsilon_{j}$, $j \in J_{2}$, corresponding information costs and best design $d$, one has to solve problem (5) parametrically, for

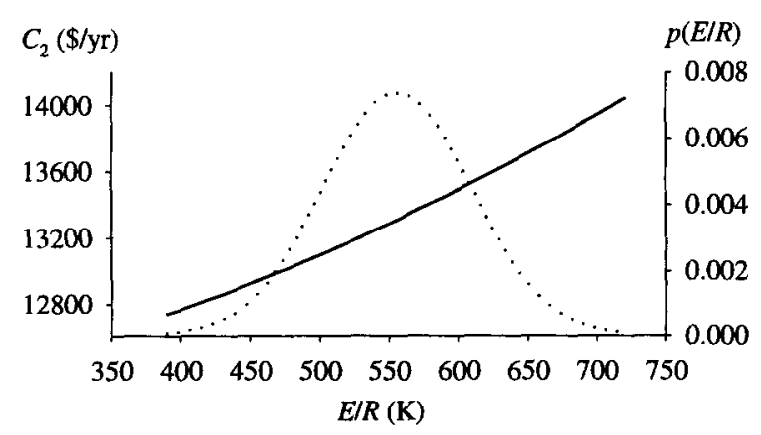

Fig. 2. Objective function values $C_{2}$ (solid line) and $E / R$ PDF (dotted line) as a function of $E / R$ true but unknown value. 
different possible realizations of vector $\mu_{j}$. Then, a possible decision criterion may be to select the worst case scenario for all possible $\mu_{j}$ true values (e.g. the point where largest optimal information costs are obtained). However, this decision may be too conservative and overestimate $R \& D$ resource allocation. Thus, one may instead want to adopt as solution the value of information costs and $R \& D$ spending that result into best average gains over the domain $\Theta_{2}=$ $\left\{\mu_{j}: \mu_{j} \in p\left(\mu_{j}\right), j \in J_{2}\right\}$, that is, to identify and pick up the bcst values for errors $\varepsilon_{j}, j \in J_{2}$, associated information costs and design $d$ that minimize the objective function expected value for process performance in face of $\mu_{j}$ uncertainty, i.e. the expected value in face of $\mu_{j}$ uncertainty of an expected value in face of $\theta$ uncertainty. Mathematically, the design problem is then formulated as follows (with the second stage decision omitted):

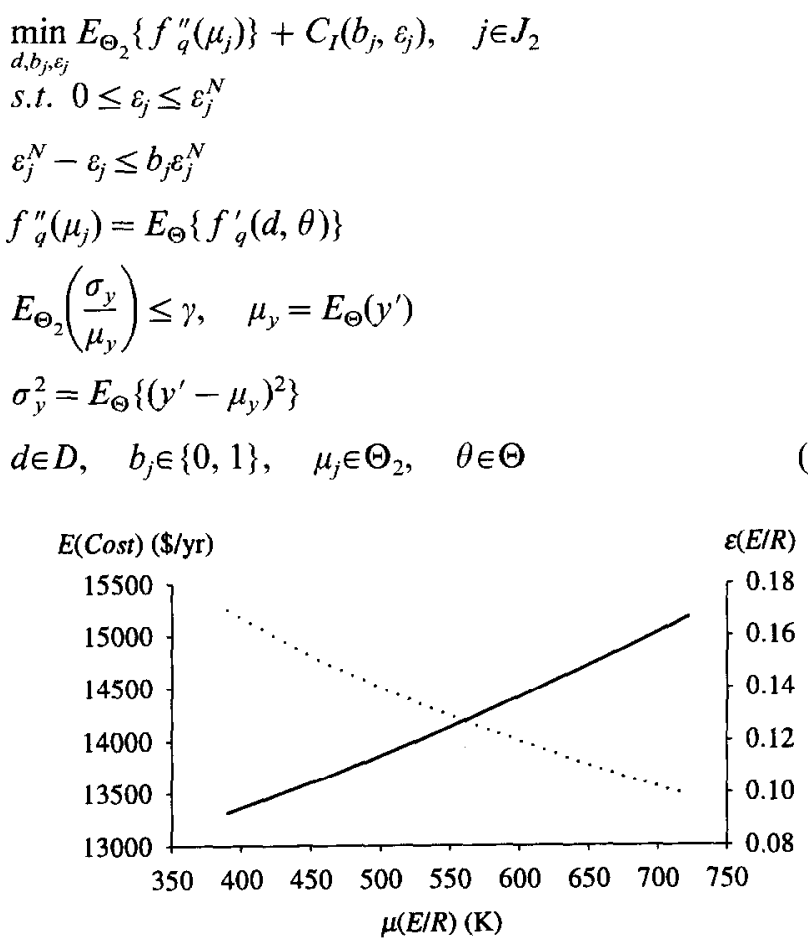

Fig. 3. Expected cost (solid line) and optimal error (dotted line) as a function of $E / R$ mean values.

Table 4

Results obtained for the worst-case scenario (C) and minimal average overall cost (D)

\begin{tabular}{lcc}
\hline & $\mathrm{C}^{\mathrm{a}}$ & $\mathrm{D}$ \\
\hline$E$ Cost (\$ per year) & 15169 & $14252^{\mathrm{b}}$ \\
$V\left(\mathrm{~m}^{3}\right)$ & 8.667 & 6.193 \\
$A\left(\mathrm{~m}^{2}\right)$ & 7.442 & 7.804 \\
$\varepsilon(E / R)$ & 0.099 & 0.133 \\
$\mu\left(x_{\mathrm{A}}\right)$ & 0.9184 & $0.925^{\mathrm{b}}$ \\
$\sigma\left(x_{\mathrm{A}}\right) / \mu\left(x_{\mathrm{A}}\right)$ & 0.01 & $0.01^{\mathrm{b}}$ \\
\hline
\end{tabular}

${ }^{\mathrm{a}} \mu(E / R)=722.3 \mathrm{~K}$.

${ }^{\mathrm{b}}$ Expected value in face of $\mu(E / R)$ uncertainty.
Going back to our case study, we now have selected activation energy as the most valuable uncertainty reduction parameter, with an expected VEU of 1279 \$ per year. In order to find out what should be the optimal investment in R\&D for gaining additional information on this parameter, we have solved problem (5) for different possible values of $\mu(E / R)$ : as the true activation energy mean increases, optimal information costs become larger, and therefore the corresponding parameter relative error, $\varepsilon(E / R)$, smaller, indicating that bigger R\&D spending should be afforded if indeed the parameter true mean value happens to correspond to the less favorable scenarios of high $E / R$ values (Fig. $3)$. The worst case scenario (C) corresponds to an optimal error of only 0.099 around the barely probable mean value of $722.3 \mathrm{~K}$ (Table 4).

A less conservative decision may be obtained using an average criterion over the domain $\Theta_{2}=\{\mu(E)$ $R): \mu(E / R) \in p[\mu(E / R)]\}$, where $p[\mu(\mathrm{E} / \mathrm{R})]$ is a normal PDF with mean $555.6 \mathrm{~K}$ and relative error 0.3 . In order to solve our problem formulation (7) for this specific example, specialized quadrature and cubature formulae were used respectively for the $\Theta_{2}$ and $\Theta$ domain integrations, resulting in a total of 228 points of evaluation. The optimal solution obtained (scenario D, Table 4) indicates that, in average, it is profitable to launch a R\&D program, with an annual depreciation value of $475 \$$ per year, in order to increasc the currently available knowledge about the activation energy, up to the point where an error of 0.133 is achieved.

\section{Conclusions}

We have developed and tested a stochastic optimization framework for achieving optimal process design under uncertainty that explores the trade-off between economic added value deriving from parameter uncertainty reduction and information costs associated with experiments and R\&D efforts needed to obtain reduced levels of uncertainty. The proposed methodology is also able to identify and screen the parameters whose uncertainty decrease will more likely lead to overall process performance gains, and for such parameters to define to what extent and at what price experiments should be conducted for achieving optimal additional information levels. This decision can be made based upon the worst case scenario or, in a less conservative way, the minimization of an overall average performance criterion over a probabilistic space of all possible true mean values.

The proposed methodology, when applied to a case study of a reactor and heat exchanger system, identified activation energy as being the key parameter in terms of additional economic value deriving from reduced parameter uncertainty levels. It was also shown that for 
this particular case it is indeed quite useful and profitable to conduct selective $R \& D$ experiments with the goal of getting additional information and knowledge about activation energy.

\section{References}

Bernardo, F. P., Pistikopoulos, E. N., \& Saraiva, P. M. (1999a). Robustness criteria in process design optimization under uncertainty. Computers \& Chemical Engineering, 23 (Suppl.), S459.

Bernardo, F. P., Pistikopoulos, E. N., \& Saraiva, P. M. (1999b). Quality costs and robustness criteria in chemical process design optimization. Computers \& Chemical Engineering, submitted for publication.
Bernardo, F. P., Pistikopoulos, E. N., \& Saraiva, P. M. (1999c). Integration and computational issues in stochastic design and planning optimization problems. Industrial \& Engineering Chemistry Research, 38, 3065.

Bernardo, F. P., \& Saraiva, P. M. (1998). Robust optimization framework for process parameter and tolerance design. American Institute of Chemical Engineering Journal, 44, 2007.

Georgiadis, M. C., \& Pistikopoulos, F. N. (1999). An integrated framework for robust and flexible process systems. Industrial \& Engineering Chemistry Research, 38, 133.

Halemane, K. P., \& Grossmann, I. E. (1983). Optimal process design under uncertainty. American Institute of Chemical Engineering Journal, 29, 425.

Ierapetritou, M. G., Pistikopoulos, E. N,, \& Floudas, C. A. (1996). Operational planning under uncertainty. Computers \& Chemical Engineering, 20, 1499.

Pistikopoulos, E. N. (1995), Uncertainty in process design and operations, Computers \& Chemical Engineering, 19 (Suppl.), S553. 